\title{
CONSTRUCTIVE TECHNIQUES IN RATIONAL APPROXIMATION
}

\author{
BY \\ T. W. GAMELIN $\left({ }^{1}\right)$ AND J. GARNETT $\left({ }^{2}\right)$
}

This paper consists of several loosely organized remarks on the constructive methods for rational approximation developed by Vitushkin in [13]. These remarks are grouped under three headings.

The first topic, taken up in $\S 1$, illustrates the simplest case of the approximation scheme, and shows how it can be used to give simple proofs of rational approximation theorems on a class of infinitely connected compact sets. Special cases of these theorems have been proved by other methods by Fisher [8] and Zalcman [16]. This section also serves as an introduction to the integral operator $T_{\phi}$, which is used in $\$ \$ 2$ and 3.

The second topic, involving pointwise bounded approximation, occupies $\S \S 2$ through 5 . In $\$ 2$, necessary and sufficient conditions are given on an open plane set $U$ in order that every bounded analytic function on $U$ be a pointwise limit on $U$ of a bounded sequence of uniformly continuous analytic functions on $U$. This result, together with Mergelyan's theorem, yields the Farrell-Rubel-Shields theorem (cf. [12]) on pointwise bounded approximation by polynomials, and its extension to finitely connected sets (cf. Corollary 3.3) by Ahern and Sarason [1]. It will be noted, however, that the constructive techniques alone do not give the best possible bounds on the norms of the approximating functions. In $\$ 3$ we obtain partial results on pointwise bounded approximation by rational functions. $\$ \$ 4$ and 5 are devoted to constructing an example of a set for which pointwise bounded approximation by uniformly continuous analytic functions obtains, whereas approximation by rational functions fails.

The final topic, relegated to $\S 6$, involves extending Vitushkin's techniques to vector-valued functions, in order to obtain results on uniform approximation by analytic functions of several complex variables. A related result has been given by Eifler in [7].

The main definitions and notations we will be using are as follows. The Riemann sphere will be denoted by $S^{2}$. If $U$ is an open subset of $S^{2}$, then $H^{\infty}(U)$ will denote the algebra of bounded analytic functions on $U$, and $A(U)$ will denote the algebra of functions continuous on the closure $\bar{U}$ of $U$ which are analytic on $U$. If $X$ is a

Received by the editors June 27, 1968.

(1) Partially supported by NSF grant GP5638.

( ${ }^{2}$ Partially supported by the Air Force Office of Scientific Research, Office of Aerospace Research, under Contract No. F44620-67-6-0029. 
compact plane set, then $C(X)$ is the algebra of all continuous complex-valued functions on $X, A(X)$ is the algebra of functions in $C(X)$ which are analytic on the interior $X^{\circ}$ of $X, R(X)$ is the algebra of functions in $C(X)$ which can be approximated uniformly on $X$ by rational functions with poles off $X$, and $P(X)$ is the algebra of functions in $C(X)$ which can be approximated uniformly on $X$ by polynomials in $z$. (With this notation notice that $A(X)$ is isomorphic to $A\left(X^{\circ}\right)$ when $X^{\circ}$ is dense in $X$.) The symbol $\|\cdot\|$ will always denote the supremum norm over the appropriate set. The closed disc with center at $z$ and radius $a$ is denoted by $\Delta(z ; a)$.

The continuous analytic capacity of a plane set $E$ is defined by

$$
\begin{aligned}
& \alpha(E)=\sup \left\{\left|f^{\prime}(\infty)\right|: f \in C\left(S^{2}\right),\|f\| \leqq 1, f\right. \text { is analytic off some } \\
& \text { compact subset of } E \text { \}. }
\end{aligned}
$$

The analytic capacity of $E$ is

$$
\gamma(E)=\sup \left\{\left|f^{\prime}(\infty)\right|: f \text { is analytic off some compact subset of } E,\|f\| \leqq 1\right\} .
$$

Evidently $\gamma$ and $\alpha$ are monotone set functions satisfying $\alpha(E) \leqq \gamma(E)$, with equality when $E$ is open. For more on analytic capacity see [13] and [15]. The inner boundary of a compact set is its boundary less the boundaries of the complementary components.

1. A basic lemma. The techniques of Vitushkin rely on functions which are constructed as in the following simple lemma (cf. [15, p. 88]).

1.1 LEMMA. Let $\phi$ be a continuously differentiable function on the plane with compact support $X$. For every bounded measurable function $f$ on the complex plane, define

$$
\left(T_{\phi} f\right)(\zeta)=\frac{1}{\pi} \iint \frac{f(z)-f(\zeta)}{z-\zeta} \frac{\partial \phi}{\partial \bar{z}} d x d y=\phi(\zeta) f(\zeta)+\frac{1}{\pi} \iint \frac{f(z)}{z-\zeta} \frac{\partial \phi}{\partial \bar{z}} d x d y
$$

Then $T_{\phi} f$ has the following properties:

(i) $T_{\phi} f$ is measurable, and

$$
\left\|T_{\phi} f\right\|_{\infty} \leqq 2 \operatorname{diam}(X)\left\|\partial \phi\left|\partial \bar{z}\left\|_{\infty} \sup _{z, \zeta \in X}|f(z)-f(\zeta)| \leqq 4 \operatorname{diam}(X)\right\| \partial \phi\right| \partial \bar{z}\right\|_{\infty}\|f\|_{X} .
$$

(ii) $T_{\phi} f$ is analytic off $X$ and vanishes at $\infty$.

(iii) $T_{\phi} f$ is continuous wherever $f$ is continuous.

(iv) $T_{\phi} f$ is analytic wherever $f$ is analytic.

(v) $f-T_{\phi} f$ is analytic on the interior of $\phi^{-1}(1)$.

Proof. The integral $\iint[f(z) /(z-\zeta)](\partial \phi / \partial \bar{z}) d x d y$, being the convolution of the locally integrable function $1 / z$ and a bounded function with compact support, depends continuously on $\zeta$. Consequently $T_{\phi} f$ is measurable, and (iii) is valid. The estimates in (i) are straightforward. Both (ii) and (v) follow immediately from the second formula for $T_{\phi} f$. 
Suppose that $f$ is analytic on a disc $\Delta$. Since $(f(z)-f(\zeta)) /(z-\zeta)$ is jointly analytic in $z$ and $\zeta$ on $\Delta \times \Delta$, the integral

$$
\iint_{\Delta} \frac{f(z)-f(\zeta)}{z-\zeta} \frac{\partial \phi}{\partial \bar{z}} d x d y
$$

is analytic on $\Delta$. Since

$$
\iint_{C \backslash \Delta} \frac{f(z)-f(\zeta)}{z-\zeta} \frac{\partial \phi}{\partial \bar{z}} d x d y
$$

is also analytic on $\Delta$, we see that $T_{\phi} f$ is analytic on $\Delta$. That proves (iv).

Lemma 1.1 provides simple proofs of many results in rational approximation. For example, it yields the following corollary (cf. Arens [2]).

1.2 Corollary. Let $f$ be a bounded measurable function on the complex plane $C$, and let $z_{0} \in C$ be fixed. Then there is a sequence of bounded measurable functions $\left\{f_{n}\right\}_{n=1}^{\infty}$ satisfying:

(i) $\left\|f_{n}\right\|_{\infty} \leqq 32\|f\|_{\infty}$.

(ii) $f_{n}$ is continuous wherever $f$ is, and analytic wherever $f$ is.

(iii) $f_{n}$ is analytic in a neighborhood of $z_{0}$.

(iv) $f_{n}(\zeta) \rightarrow f(\zeta)$ for all complex $\zeta \neq z_{0}$.

Moreover, if $f$ is continuous at $z_{0}$, then

(iv)' $f_{n}$ converges uniformly to $f$.

Proof. Let $\phi_{n}$ be a continuously differentiable function such that $\phi_{n}(z)=0$ when $\left|z-z_{0}\right| \geqq 2 / n, \phi_{n}(z)=1$ when $\left|z-z_{0}\right| \leqq 1 / n$, and $\left\|\partial \phi_{n} / \partial \bar{z}\right\|_{\infty} \leqq 4 n$. Then the $T_{\phi_{n}} f$ form a bounded sequence. A normal families argument shows that the $T_{\phi_{n}} f$ converge pointwise to zero, except at $z_{0}$. If $f$ is continuous at $z_{0}$, the convergence is uniform. The desired sequence is then $f_{n}=f-T_{\phi_{n}} f$.

1.3 Corollary. Let $X$ be a compact plane set obtained from the closed unit disc by deleting a sequence of pairwise disjoint open discs which cluster at only finitely many points. If $f \in H^{\infty}\left(X^{\circ}\right)$, there is a bounded sequence $\left\{f_{n}\right\}_{n=1}^{\infty}$ in $R(X)$ such that $f_{n}(z) \rightarrow f(z)$ for all $z \in X^{\circ}$. If $f \in A(X)$, there is a sequence $\left\{f_{n}\right\}_{n=1}^{\infty}$ in $R(X)$ such that $f_{n} \rightarrow f$ uniformly on $X$. (That is, $R(X)=A(X)$.)

Proof. In each case, 1.2 reduces the problem to a set whose complement has finitely many components, where the result is known (and easy to prove, in this special case).

There is an equivalent dual version of 1.1 used to prove 1.3 in [15, p. 123]. Since $T_{\phi}$ maps $C(X)$ into $C\left(S^{2}\right)$, its adjoint $T_{\phi}^{*}$ maps the measures on $S^{2}$ into the measures on $X$. It is easy to see that $T_{\phi}^{*}$ has the form

$$
T_{\phi}^{*} \mu=\phi \mu-\frac{1}{\pi} \frac{\partial \phi}{\partial \bar{z}} \hat{\mu}(z) d x d y
$$


where $\hat{\mu}(z)$ is the Cauchy transform

$$
\hat{\mu}(z)=\int \frac{d \mu(\zeta)}{z-\zeta}
$$

The key to the "dual" lemma is that

$$
\left(T_{\phi}^{*} \mu\right)^{\wedge}=\phi \cdot \hat{\mu} .
$$

See [10] for a proof.

2. Bounded pointwise approximation. In this section we ask, for an open set $U$, when $A(U)$ is boundedly pointwise dense in $H^{\infty}(U)$, that is, when is each $f \in H^{\infty}(U)$ the pointwise limit on $U$ of a sequence $\left\{f_{n}\right\}_{n=1}^{\infty}$ in $A(U)$ satisfying $\left\|f_{n}\right\| \leqq c\|f\|$. For convenience we always assume $\infty \notin \partial U$. A routine category argument shows that the constant $c$ depends only on $U$. It would be of interest to know when the constant $c$ can be taken to be 1 , but here we give no information on that score.

We begin with a localization lemma akin to Bishop's theorem (11.8 of [15]).

2.1 Lemma. Let $U$ be an open subset of $S^{2}$ with $\infty \notin \partial U$. Let $f \in H^{\infty}(U)$. Suppose there is an open cover $\left\{U_{j}\right\}_{j=1}^{n}$ of $\partial U$ such that on each set $U \cap U_{j}, f$ is the pointwise limit of a sequence $\left\{f_{m}\right\}_{m=1}^{\infty}$ in $A\left(U \cap U_{j}\right)$ satisfying

$$
\left\|f_{m}\right\|_{U \cap U_{j}} \leqq M\|f\|_{U \cap U_{j}} .
$$

Then $f$ can be approximated pointwise on $U$ by a sequence $\left\{g_{m}\right\}_{m=1}^{\infty}$ in $A(U)$ satisfying

$$
\left\|g_{m}\right\|_{U} \leqq \lambda \cdot M\|f\|_{U}
$$

where $\lambda$ depends only on the cover $\left\{U_{j}\right\}$.

Proof. Let $\left\{\phi_{j}\right\}_{j=1}^{n}$ be infinitely differentiable functions such that the closed support of $\phi_{j}$ is a compact subset of $U_{j}$, and $\sum \phi_{j}=1$ near $\partial U$. Set $f=0$ off $U$ and let $f_{j}=T_{\phi_{j}}(f)$. Using 1.1 , we see that $f-\sum f_{j}$ is entire and tends to the limit $f(\infty)$ at $\infty$. By Liouville's theorem

$$
f=f(\infty)+\sum f_{j}
$$

Let $\left\{h_{j, m}\right\}_{m=1}^{\infty}$ be a sequence in $A\left(U \cap U_{j}\right)$ such that $\left\|h_{j, m}\right\| \leqq M\|f\|$, and $h_{j, m}(z)$ $\rightarrow f(z)$ for $z \in U \cap U_{j}$. We can assume that $h_{j, m} \in C\left(S^{2}\right)$, that $h_{j, m} \rightarrow 0$ off $\bar{U}$, and that $h_{j, m} \rightarrow h_{j}$ in the weak-star topology of $L^{\infty}(d x d y)$. Set $g_{j, m}=T_{\phi_{j}}\left(h_{j, m}\right)$ and set $G_{m}=\sum_{j} g_{j, m}$. Using 1.1 , we find that $G_{m} \in A(U)$, and that $\left\|G_{m}\right\| \leqq \lambda_{1} M\|f\|$, where $\lambda_{1}$ depends only on $\left\{\phi_{j}\right\}_{j=1}^{n}$.

If $\xi \in U$, then $\phi_{j}(\xi) h_{j, m}(\xi) \rightarrow \phi_{j}(\xi) f(\xi)$. Recalling the definition of $T_{\phi}$, we have

$$
\begin{aligned}
\lim _{m \rightarrow \infty} g_{j, m}(\xi)= & \phi_{j}(\xi) f(\xi)+\frac{1}{\pi} \iint_{U} \frac{f(z)}{z-\xi} \frac{\partial \phi_{j}}{\partial \bar{z}} d x d y \\
& +\frac{1}{\pi} \iint_{\partial U} \frac{h_{j}(z)}{z-\xi} \frac{\partial \phi_{j}}{\partial \bar{z}} d x d y \\
= & f_{j}(\xi)+H_{j}(\xi),
\end{aligned}
$$


where

$$
H_{j}(\xi)=\frac{1}{\pi} \iint_{\partial U} \frac{h_{j}(z)}{z-\xi} \frac{\partial \phi_{j}}{\partial \bar{z}} d x d y .
$$

Now $H_{j}$ is the convolution of a compactly supported bounded measurable function and the locally integrable function $1 / z$. So $H_{j} \in C\left(S^{2}\right)$. Also $H_{j}$ is analytic off $\partial U$, whence $H_{j} \in A(U)$. Since $\left\|h_{j}\right\| \leqq \lim \sup \left\|h_{j m}\right\| \leqq M\|f\|$, we obtain $\left\|H_{j}\right\| \leqq \lambda_{2} M\|f\|$, where $\lambda_{2}$ depends only on $\left\{\phi_{j}\right\}$.

Now set

$$
F_{m}=f(\infty)+G_{m}-\sum_{j=1}^{n} H_{j}
$$

Then $F_{m} \in A(U)$ satisfies $\left\|F_{m}\right\| \leqq \lambda M\|f\|$, where $\lambda$ depends only on $\left\{\phi_{j}\right\}_{j=1}^{n}$. And

$$
F_{m}(\xi) \rightarrow f(\infty)+\sum f_{j}(\xi)=f(\xi)
$$

for all $\xi \in U$.

A glance at the functions estimated shows that we may take

$$
\begin{aligned}
\lambda & =1+\frac{2}{\pi} \sum_{j=1}^{n} \sup _{\xi \in C} \iint\left|\frac{\partial \phi_{j}}{\partial \bar{z}}\right| \frac{1}{|z-\xi|} d x d y \\
& \leqq 1+2 \sum_{j=1}^{n} \operatorname{diam}\left(X_{j}\right)\left\|\frac{\partial \phi_{j}}{\partial \bar{z}}\right\|,
\end{aligned}
$$

where $X_{j}$ is the support of $\partial \phi_{j} / \partial \bar{z}$. This remark will be used in the proof of the next theorem.

2.2 TheOREM. Let $U$ be an open subset of $S^{2}$ such that $\infty \notin \partial U$. Then the following are equivalent:

(i) $A(U)$ is pointwise boundedly dense in $H^{\infty}(U)$.

(ii) For each $r>1$, there exists $c>0$ such that

$$
\gamma(\Delta(z ; \delta) \backslash U) \leqq c \alpha(\Delta(z ; r \delta) \backslash U)
$$

for all $\delta>0$ and all complex $z$.

(iii) There exists $r>1$ and $c>0$ such that for all $\delta>0$ sufficiently small,

$$
\gamma(\Delta(z ; \delta) \cap \partial U) \leqq c \alpha(\Delta(z ; r \delta) \backslash U), \quad z \in \partial U .
$$

Proof. First we use the localization lemma to show that (i) implies (ii). For this suppose that every $f \in H^{\infty}(U)$ is the pointwise limit on $U$ of a sequence $\left\{f_{n}\right\}_{n=1}^{\infty}$ in $A(U)$ satisfying $\left\|f_{n}^{\prime}\right\| \leqq M\|f\|$. Fix $\delta>0, r>1$, and a complex number $z_{0}$. By the definition of $\gamma$, there is a function $g$ analytic on $U \cup\left\{\left|z-z_{0}\right|>\delta\right\}$, such that $|g| \leqq 1$ and

$$
2\left|g^{\prime}(\infty)\right| \geqq \gamma\left(\Delta\left(z_{0} ; \delta\right) \mid U\right) .
$$

We will apply 2.1 to the function $g$ and the set $V=U \cup\left\{\left|z-z_{0}\right|>r \delta\right\}$. 
The open sets $V_{1}=\left\{\left|z-z_{0}\right|>\delta\right\}$ and $V_{2}=\left\{\left|z-z_{0}\right|<r \delta\right\}$ cover $\partial V$. The functions $g((1+1 / n) z) \in A\left(V_{1}\right)$ are bounded by 1 and converge to $g$ pointwise on $V_{1}$. Since $V_{2} \cap V \subset U$, and since $g \in H^{\infty}(U), g$ is approximable pointwise on $V_{2} \cap V$ by functions in $A\left(V_{2} \cap V\right)$ where norms do not exceed $M$. By 2.1, there is a sequence $\left\{g_{n}\right\}_{n=1}^{\infty}$ on $A(V)$ converging pointwise to $g$ on $V$, such that $\left\|g_{n}\right\| \leqq \lambda M$. We can assume $g_{n} \in C\left(S^{2}\right)$. Then

$$
\left|g_{n}^{\prime}(\infty)\right| \leqq\left\|g_{n}\right\| \alpha\left(\Delta\left(z_{0} ; r \delta\right) \backslash U\right) .
$$

Since $g_{n}^{\prime}(\infty) \rightarrow g^{\prime}(\infty)$, we obtain

$$
\gamma\left(\Delta\left(z_{0} ; \delta\right) \backslash U\right) \leqq 2 \lambda M \alpha\left(\Delta\left(z_{0} ; r \delta\right) \backslash U\right) .
$$

It remains now to check that $\lambda$ is independent of $\delta$ and $z_{0}$. Let $\phi_{1}$ be a continuously differentiable function such that $\phi_{1} \equiv 0$ near $\Delta\left(z_{0} ; \delta\right), \phi_{1} \equiv 1$ near $\left\{\left|z-z_{0}\right|=r \delta\right\}$, $\phi_{1} \equiv 0$ for $\left|z-z_{0}\right| \geqq 2 r \delta$, and $\left|\partial \phi_{1} / \partial \bar{z}\right| \leqq 4 /(r-1) \delta$. Set $\phi_{2} \equiv 0$ if $\left|z-z_{0}\right| \geqq r \delta$, and $\phi_{2}=1-\phi_{1}$ on $\Delta\left(z_{0} ; r \delta\right)$. Then $\phi_{j}$ is supported inside $V_{j}$, and $\phi_{1}+\phi_{2}=1$ on $\partial V$. The support of $\phi_{j}$ has diameter not exceeding $4 r \delta$. By the remark following 1.1, we can take

$$
\lambda=1+64 r /(r-1)
$$

This shows (ii) holds with

$$
c=2 M(1+64 r /(r-1)) .
$$

That (ii) implies (iii) holds because $\gamma$ is monotone.

That (iii) implies (i) follows from the techniques developed by Vitushkin. We give a sketch of the proof, to show how Vitushkin's arguments can be modified to obtain the result.

So suppose that (iii) is true, and that $f \in H^{\infty}(U)$. For each fixed $\delta>0$, choose points $z_{\kappa \delta}$ and continuously differentiable functions $\phi_{\kappa \delta}$ such that

(i) $z_{\kappa \delta} \in \partial U$.

(ii) $\phi_{\kappa \delta}$ is supported on $\Delta\left(z_{\kappa \delta} ; \delta\right)$.

(iii) $\sum \phi_{\kappa \delta}=1$ in a neighborhood of $\partial U$.

(iv) $\left\|\partial \phi_{\kappa \delta} / \partial \bar{z}\right\|_{\infty} \leqq 4 / \delta$.

(v) No complex number $z$ is contained in more than $M$ of the discs $\Delta\left(z_{\kappa \delta} ; \delta\right)$, where $M$ is a universal constant.

Set $f=0$ off $U$, and define $f_{\kappa \delta}=T_{\phi_{\kappa \delta}}(f)$. Then

Applying 1.1, we find that

$$
f=f(\infty)+\sum_{\kappa} f_{\kappa \delta}
$$

$$
\left\|f_{\kappa \delta}\right\| \leqq 32\|f\|, \quad\left|f_{\kappa \delta}^{\prime}(\infty)\right| \leqq 32\|f\|_{\infty} \gamma\left(\Delta\left(z_{\kappa} ; \delta\right) \cap \partial U\right) .
$$

Now Vitushkin's analysis of the situation (cf. [12], especially $\S I V .3$ and IV.4, where the argument must be modified merely by replacing the modulus of continuity of $f$ by $\|f\|)$ together with our hypothesis, produce functions $F_{\kappa \delta} \in C\left(S^{2}\right)$ 
such that $F_{\kappa \delta}$ is analytic off $\Delta\left(z_{\kappa \delta} ; r \delta\right) \backslash U, F_{\kappa \delta}-f_{\kappa \delta}$, has a triple zero at $\infty$, and $\left\|F_{\kappa \delta}\right\| \leqq b\|f\|$, where the constant $b$ depends only on $c$ and $r$, but not on $\delta$.

Now $\left\|f_{\kappa \delta}-F_{\kappa \delta}\right\| \leqq b\|f\|+32\|f\|=a\|f\|$. Since $\left(z-z_{\kappa \delta}\right)^{3}\left(F_{\kappa \delta}-f_{\kappa \delta}\right)$ is analytic outside $\Delta\left(z_{\kappa \delta} ; r \delta\right)$ and at $\infty$, and since it is bounded by $a r^{3} \delta^{3}\|f\|$ on the boundary of $\Delta\left(z_{\kappa \delta} ; r \delta\right)$, we obtain

$$
\left|f_{\kappa \delta}(z)-F_{\kappa \delta}(z)\right| \leqq a r^{3} \delta^{3}\|f\| /\left|z-z_{\kappa \delta}\right|^{3}
$$

whenever $\left|z-z_{\kappa \delta}\right| \geqq r \delta$. This estimate continues to hold for all complex $z$.

Now fix the complex number $z \in \bar{U}, z \neq \infty$. Let $N(n)$ be the number of discs $\Delta\left(z_{\kappa \delta} ; \delta\right)$ which touch the circle $\partial \Delta(z ; n \delta)$. Since each complex number is contained in at most $M$ of the discs $\Delta\left(z_{\kappa \delta} ; \delta\right)$, an elementary argument involving areas shows that $N(n) \leqq 4 M n$. If $n \geqq 2$, and $\Delta\left(z_{\kappa \delta} ; \delta\right)$ touches the $n$th circle, then $\left|z-z_{\kappa \delta}\right| \geqq(n-1) \delta$ and $\left|f_{\kappa \delta}(z)-F_{\kappa \delta}(z)\right| \leqq r^{3} a\|f\| /(n-1)^{3}$. Using the estimate $\left|f_{\kappa \delta}(z)-F_{\kappa \delta}(z)\right| \leqq a\|f\|$ if $\Delta\left(z_{\kappa \delta} ; \delta\right)$ meets the first circle, we obtain

$$
\begin{aligned}
\left|f(z)-f(\infty)-\sum F_{\kappa \delta}(z)\right| & \leqq \sum_{\kappa}\left|f_{\kappa \delta}(z)-F_{\kappa \delta}(z)\right| \\
& \leqq a\|f\|\left(N(1)+\sum_{n=2}^{\infty} r^{3} N(n) /(n-1)^{3}\right) .
\end{aligned}
$$

Set $f_{\delta}=f(\infty)+\sum_{\kappa} F_{\kappa \delta} \in A(U)$. The preceding estimate shows that the $f_{\delta}$ are uniformly bounded as $\delta \rightarrow 0$.

Suppose $z \in U$. Let $d$ be the distance from $z$ to $\partial U$, and let $[d / \delta]$ be the largest integer not exceeding $d / \delta$. Then $N(n)=0$ when $(n+1) \delta<d$, so

$$
\left|f(z)-f_{\delta}(z)\right| \leqq 4 a\|f\| M r^{3} \sum_{n=[d / \delta]}^{\infty} n /(n-1)^{3} .
$$

Since $[d / \delta] \rightarrow \infty$ as $\delta \rightarrow 0$, we obtain $f_{\delta}(z) \rightarrow f(z)$ as $\delta \rightarrow 0$. Hence (iii) implies (i). That completes the proof.

Now we turn to the special case, in which the diameter of every component of $\partial U$ exceeds some constant $d>0$. In this case, $\Delta(z ; \delta) \backslash U$ contains a connected set of diameter $\delta$ whenever $\delta \leqq d$ and $z \in \partial U$. By an elementary estimate of analytic capacity (essentially Koebe's $1 / 4$-theorem, cf. [15, p. 13]) we have $\gamma(\Delta(z ; \delta) \mid U) \geqq \delta / 4$ for $z \in \partial U$ and $\delta \leqq d$. Since $\gamma(\Delta(z ; \delta) \backslash U) \leqq \delta$ always, we can rephrase 2.2 as follows.

2.3 CoROllary. Suppose that the diameters of the components of $\partial U$ are bounded away from zero. Then $A(U)$ is pointwise boundedly dense in $H^{\infty}(U)$ if and only if there exists $a>0$ such that for all $\delta>0$ sufficiently small,

$$
\alpha(\Delta(z ; \delta) \backslash U) \geqq a \delta, \quad z \in \partial U .
$$

An example for which $\partial U$ is connected but for which approximation does not occur appears at the end of $\S 3$.

One relation between pointwise bounded approximation and an abstract function algebraic concept is contained in the following corollary. For a discussion of peak points see [15]. 
2.4 Corollary. Suppose that the diameters of the components of $\partial U$ are bounded away from zero. If $A(U)$ is boundedly pointwise dense in $H^{\infty}(U)$, then every point of $\partial U$ is a peak point for $A(U)$.

Proof. The analogue of Curtis's criterion (cf. [4]) for $A(U)$, regarded as a subalgebra of $C(\bar{U})$, states that $z_{0} \in \partial U$ is a peak point for $A(U)$ whenever

$$
\limsup _{\delta \rightarrow 0} \frac{\alpha\left(\Delta\left(z_{0} ; \delta\right) \mid U\right)}{\delta}>0 .
$$

By 2.3, this condition is fulfilled at every point of $\partial U$.

It is conjectured that the converse of 2.4 is also valid. Another related conjecture is that $A(U)$ is a dirichlet algebra on $\partial U$ whenever $\partial U$ is connected and every point of $\partial U$ is a peak point for $A(U)$. A special case of this conjecture is proved in $\S 4$.

For certain special sets $U$, it can be shown that condition (i) of 2.2 is equivalent to the following:

(i) The unit ball of $A(U)$ is pointwise boundedly dense in the unit ball of $H^{\infty}(U)$. See [9] for details. It is not known whether (i)' is always equivalent to (i). An affirmative answer would show that $A(K)$ is pointwise boundedly dense in $H^{\infty}\left(K^{\circ}\right)$ whenever the inner boundary of $K$ is at most countable. An affirmative answer would also show that (i)-(iii) are equivalent to the following pointwise condition:

(iv) For each $z \in \partial U$, there exists $r>1$ such that

$$
\limsup _{\delta \rightarrow 0} \frac{\gamma(\Delta(z ; \delta) \cap \partial U)}{\alpha(\Delta(z ; r \delta) \backslash U)}<\infty .
$$

3. Pointwise bounded approximation by $R(K)$. Let $K$ be a compact plane set. Then $A(K)$ consists of the functions on $A\left(K^{\circ}\right)$ extended in all possible continuous ways to $K$. So the preceding section gives necessary and sufficient criteria that $A(K)$ be boundedly pointwise dense in $H^{\infty}\left(K^{\circ}\right)$. The corresponding problem for $R(K)$ seems to be more difficult, and we have not been able to solve it in general. However, the arguments of $\$ 2$ can be carried over to give some partial results. The analogue of the localization lemma is the following.

3.1 Lemma. Let $K$ be a compact subset of $S^{2}$ such that $\infty \notin \partial K$, and such that $\partial K$ has zero area. Let $f \in H^{\infty}\left(K^{\circ}\right)$. Suppose there is an open cover $\left\{U_{j}\right\}_{j=1}^{n}$ of $\partial K^{\circ}$ such that on each $K^{\circ} \cap U_{j}, f$ is the pointwise limit of a sequence $f_{m}$ in $R\left(K \cap \bar{U}_{j}\right)$ satisfying $\left\|f_{m}\right\| \leqq M\|f\|$. Then $f$ can be approximated pointwise on $K^{\circ}$ by a sequence $g_{m}$ in $R(K)$ satisfying $\left\|g_{m}\right\| \leqq c M\|f\|$, where $c$ depends only on the cover $\left\{U_{j}\right\}$.

Proof. Note that in this case the function $G_{m}$ in the proof of 2.1 is analytic in a neighborhood of $K$, while the $H_{j}$ vanish since $\partial K$ has zero area. With these remarks, the proof of 2.1 applies.

3.2 THEOREM. Let $K$ be a compact plane set. If there exist $r>1$ and $c>0$ such that

$$
\gamma\left(\Delta(z ; \delta) \cap \partial K^{\circ}\right) \leqq c \gamma(\Delta(z ; \delta) \backslash K)
$$


for $\delta>0$ sufficiently small and all $z \in \partial K^{\circ}$, then $R(K)$ is pointwise boundedly dense in $H^{\infty}\left(K^{\circ}\right)$. Conversely, if $\partial K$ has zero area, and if $R(K)$ is pointwise boundedly dense in $H^{\infty}\left(K^{\circ}\right)$, then for each $r>1$, there exists $c>0$ such that

$$
\gamma\left(\Delta(z ; \delta) \backslash K^{\circ}\right) \leqq c \gamma(\Delta(z ; r \delta) \backslash K)
$$

for all $\delta>0$ and all complex $z$.

3.3 Corollary. Let $K$ be a compact plane set such that the diameters of the components of $K^{c}$ are bounded away from zero. Then $R(K)$ is pointwise boundedly dense in $H^{\infty}\left(K^{\circ}\right)$.

Proof. In this case, $\gamma(\Delta(z ; r \delta) \backslash K) \geqq r \delta / 4$ providing $\delta$ is sufficiently small, and $z \in \partial K$. Since always $\gamma\left(\Delta(z ; \delta) \cap \partial K^{\circ}\right) \leqq \delta$, we obtain the estimate required to apply 3.2 .

Note that 3.3 also follows from 2.3 and the fact that $R(K)=A(K)$ (cf. [15, p. 125]).

3.4 COROLlaRY. If $\partial K$ has zero area and $R(K)$ is boundedly pointwise dense in $H^{\infty}\left(K^{\circ}\right)$, then $R(K)=A(K)$.

Proof. Let $z \in \partial K$. Since $\gamma$ dominates $\alpha$ while $\gamma(V)=\alpha(V)$ for all open $V$, we have by 3.2

$$
\alpha\left(\Delta(z, \delta) \backslash K^{\circ}\right) \leqq c \alpha(\Delta(z, r \delta) \backslash K) .
$$

This is Vitushkin's condition that $R(K)=A(K)$.

The hypothesis that $\partial K$ has zero area is unnecessarily stringent, but we are unable to weaken it substantially. It suffices to assume, for instance, that the set of points on $\partial K$ which are not peak points for $R(K)$ have zero area. We suspect that the sufficient condition in 3.1 (or a related condition) is both necessary and sufficient. We also conjecture that 3.3 holds with the hypothesis $R(\partial K)=C(\partial K)$.

The inequivalence of bounded pointwise approximation and uniform approximation is exhibited by the following example, essentially due to S. Fisher [8].

EXAMPLE. Let $\Delta$ be the closed unit disc and let $\left\{\Delta^{\circ}\left(a_{n} ; r_{n}\right)\right\}$ be a sequence of pointwise disjoint open discs in $\Delta$ whose boundaries touch and are dense on the interval $[0,1]$, and which satisfy $\sum r_{n}<1$. Set

$$
K=\Delta \backslash \bigcup_{n=1}^{\infty} \Delta^{\circ}\left(a_{n} ; r_{n}\right)
$$

Then $\partial K$ is connected and has zero area. The algebras $R(K)$ and $A(K)$ coincide because the inner boundary of $K$ lies on a line segment [13, p. 185], while the bounded analytic function

$$
F(z)=\exp \left(i \int_{0}^{1} \frac{d x}{x-z}\right)
$$

is not a pointwise bounded limit of functions in $R(K)$. A related more detailed example appears in $\$ 5$. 
4. Construction of some dirichlet algebras. Let $B$ be a uniform algebra on a compact space $X$, that is, $B$ is a closed separating subalgebra of $C(X)$ which contains the constants. The algebra $B$ is dirichlet on $X$ if the space $\operatorname{Re}(B)$ of real parts of functions in $B$ is dense in $C_{R}(X)$. For information on dirichlet algebras, see [14].

Let $K$ be a compact subset of the Riemann sphere $S^{2}$, and let $B_{K}$ be the algebra of functions in $C(K)$ which can be extended continuously to $S^{2}$ so as to be analytic on $S^{2} \mid K$. Notice that $B_{K}$ consists of the functions in $A\left(S^{2} \mid K\right)$, extended in all possible continuous ways to $S^{2}$.

The compact set $K$ is a dirichlet set if $B_{K}$ is a dirichlet algebra on $K$. If $K$ is a dirichlet set, then $K$ is connected. In the other direction, Walsh's theorem shows that if $K^{\circ}$ is connected and dense in $K$, then $K$ is a dirichlet set. More generally, if $K^{\circ}$ has a finite number of components, $K^{\circ}$ is dense in $K$, and $K$ is connected, then $K$ is a dirichlet set (cf. [1], [11]).

In this section, we will construct some new dirichlet algebras from old ones by proving that, under certain hypotheses, the union of two dirichlet sets is a dirichlet set. We suspect that the union of two dirichlet sets which meet is always a dirichlet set. The first lemma is a very special case of this conjecture, which will illustrate the techniques involved which we will be using. We will assume familiarity with the notion of "sweeping" measures to the boundary, using harmonic measure (cf. [11]).

4.1 LeMmA. If $K$ is a dirichlet set, and $J$ is a closed disc which meets $K$ at precisely one point, then $K \cup J$ is a dirichlet set.

Proof. The proof will be based on the following theorem of Glicksberg [11]: If $K_{1}$ and $K_{2}$ are disjoint dirichlet sets, then the closure of $\operatorname{Re}\left(B_{K_{1} \cup K_{2}}\right)$ has codimension one in $C_{R}\left(K_{1} \cup K_{2}\right)$. The real functionals orthogonal to $\operatorname{Re}\left(B_{K_{1} \cup K_{2}}\right)$ are the multiples of the "conjugate period" functional $u \rightarrow \int_{\Gamma}^{*} d \tilde{u}$, where $\tilde{u}$ is the harmonic extension of $u \in C_{R}\left(K_{1} \cup K_{2}\right)$ to $S^{2}$, and $\Gamma$ is a simple closed Jordan curve separating $K_{1}$ and $K_{2}$. By reducing to the case of an annulus, the norm of the "conjugate period" functional is computed to be $4 \pi /|\log r|$, where $r$ is the conformal radius of the annulus between $K_{1}$ and $K_{2}$.

Suppose now that $K \cap J=\left\{z_{0}\right\}$, so that $z_{0} \in \partial J$. Let $\left\{J_{n}\right\}_{n=1}^{\infty}$ be an increasing sequence of closed Jordan domains such that $J_{n} \subseteq J, z_{0} \notin J_{n}$, and

$$
J_{n} \cap\left\{\left|z-z_{0}\right| \geqq 1 / n\right\}=J \cap\left\{\left|z-z_{0}\right| \geqq 1 / n\right\} .
$$

(For instance, we could take $J_{n}=J \cap\left\{\left|z-z_{0}\right| \geqq 1 / n\right\}$.)

Let $\nu_{n}$ be the real measure on $\partial K \cup \partial J_{n}$ which represents the conjugate period functional orthogonal to $B_{K \cup J_{n}}$. If $m>n$, then the sweep of $\nu_{m}$ to $\partial K \cap \partial J_{n}$ is $\nu_{n}$. In particular, $\left\|\nu_{m}\right\| \geqq\left\|\nu_{n}\right\|$ if $m \geqq n$.

Suppose that $\left\|\nu_{n}\right\|$ remains bounded. Let $r_{m}$ be the conformal radius of the annulus between $K$ and $J_{n}$. Then $r_{n} \rightarrow r$ where $r<1$. Let $f_{n}$ be the conformal 
mapping of the annulus $\left\{r_{n}<|z|<1\right\}$ onto $U \backslash J_{n}$ where $U$ is the component of $S^{2} \mid K$ containing $J$. We can suppose that $f_{n} \rightarrow f$ uniformly on compact subsets of $\{r<|z|<1\}$. Elementary complex analysis shows that $f$ is a conformal map of $\{r<|z|<1\}$ onto $U \backslash J$. However $U \backslash J$ is simply connected. This contradiction shows that

$$
\lim _{n \rightarrow \infty}\left\|\nu_{n}\right\|=\infty
$$

Now let $\mu$ be a real measure on $K \cup J$ which is orthogonal to $B_{K \cup J}$, and let $\mu_{n}$ be the sweep of $\mu$ to $K \cup J_{n}$. Since the only mass of $\mu$ which is being swept lies on $J \backslash J_{n}$, we have

$$
\left\|\mu-\mu_{n}\right\| \leqq|\mu|\left(\left\{0<\left|z-z_{0}\right|<1 / n\right\}\right) \rightarrow 0 .
$$

Now $\mu_{n}$ is a real measure on $K \cup J_{n}$ orthogonal to $B_{K \cup J_{n}}$, so $\mu_{n}=c_{n} \nu_{n}$ for some constant $c_{n}$. If $m>n$, the sweeps of $\mu_{m}$ and $\nu_{m} \partial K \cup \partial J_{n}$ are $\mu_{n}$ and $\nu_{n}$ respectively. Consequently the constants $c_{n}$ coincide. Since $\left\|v_{n}\right\| \rightarrow+\infty$, we have $c_{n}=0$ for all $n$, and $\mu_{n}=0$. Consequently $\mu=0$. It follows that $K \cup J$ is a dirichlet set.

The same methods can be sharpened to prove the following generalization of 4.1.

4.2 THEOREM. Let $J$ be a closed Jordan domain ( $\partial J$ is a simple closed Jordan curve) on $S^{2}$. If $K$ is any dirichlet set which meets $J$, then $K \cup J$ is a dirichlet set.

Proof. For the sake of definiteness, we assume that there are an infinite number of component $\operatorname{arcs}\left\{\Gamma_{n}\right\}_{n=1}^{\infty}$ of $(\partial J) \backslash K$. Let $\left\{E_{n}\right\}_{n=1}^{\infty}$ be disjoint closed Jordan domains such that $\Gamma_{n} \subseteq E_{n} \subseteq J$, and $E_{n}$ meets $K$ only at the two endpoints of $\Gamma_{n}$. It is easy to see that the disc of 4.1 can be replaced by a closed Jordan domain which meets $K$ in two points. Using this observation, and induction, we find that $K \cup E_{1} \cup \ldots \cup E_{m}$ is a dirichlet set for any integer $m$.

Let $\mu$ be a real measure on $K \cup J$ orthogonal to $B_{K \cup J}$. Then $\mu$ is supported on $K \cup \partial J$. Let $\mu_{m}$ be the sweep of $\mu$ to $\partial\left(K \cup E_{1} \cup \cdots \cup E_{m}\right)$. The mass of $\mu$ being swept lies on $\bigcup_{k=m+1}^{\infty} \Gamma_{k}$, so

$$
\left\|\mu-\mu_{m}\right\| \leqq|\mu|\left(\bigcup_{k=m}^{\infty} \Gamma_{K}\right) \rightarrow 0 .
$$

However, $\mu_{m}$ is a real measure orthogonal to $B_{K \cup E_{1} \cup \cdots \cup E_{m}}$. So $\mu_{m}=0, \mu=0$, and $K \cup J$ is dirichlet.

The same technique of sweeping and passing to the limit proves the following.

4.3 THEOREM. Let $K$ be a dirichlet set. Let $\left\{J_{n}\right\}_{n=1}^{\infty}$ be closed Jordan domains such that each $J_{n}$ meets $K$, and $K \cup\left(\bigcup_{n=1}^{\infty} J_{n}\right)$ is closed. Then $K \cup\left(\bigcup_{n=1}^{\infty} J_{n}\right)$ is a dirichlet set.

5. An example. According to Browder and Wermer [3], there is an arc $\Gamma$ on $S^{2}$ which is a dirichlet set. We will obtain our example by modifying this arc according to an idea of Dolzhenko [5]. 
We can suppose that $\infty \notin \Gamma$. Let $\left\{\Delta_{j}\right\}_{j=0}^{\infty}$ be a sequence of open discs with disjoint closures, such that $\infty \in \Delta_{0}$, the radii of the $\Delta_{j}$ tend to zero, each $\bar{\Delta}_{j}$ touches $\Gamma$ at precisely one point, and $\bigcup_{j=0}^{\infty} \Delta_{j}$ is dense in $\Gamma$. Let $X=S^{2} \backslash\left(\bigcup_{j=0}^{\infty} \Delta_{j}\right)$, then:

(1) $X$ is a compact plane set.

(2) $X^{\circ}$ is connected and simply connected.

(3) $X^{\circ}$ is dense in $X$.

By 4.3, $K=\Gamma \cup\left(\bigcup_{j=0}^{\infty} \bar{\Delta}_{j}\right)$ is a dirichlet set. The algebra $B_{K}$ consists of the functions in $A(X)$, extended in all possible continuous ways to $S^{2}$. Consequently

(4) $A(X)$ is a dirichlet algebra on $\partial X$.

From the theory of dirichlet algebras, we have the following (cf. [14]).

(5) If $f \in H^{\infty}\left(X^{\circ}\right)$, there is a sequence $\left\{f_{n}\right\}_{n=1}^{\infty}$ in $A(X)$ such that $\left\|f_{n}\right\|_{X} \leqq\|f\|_{\infty}$ and $f_{n}(z) \rightarrow f(z)$ for all $z \in X^{\circ}$.

Now suppose, in addition, that the radii $r_{j}$ of the $\Delta_{j}$ are chosen so that $\sum_{j=1}^{\infty} r_{j}<\infty$. Let $\Gamma_{j}$ be a closed curve obtained by pushing $\partial \Delta_{j}$ slightly into $X^{\circ}$, except at the point where $\partial \Delta_{j}$ meets $\Gamma$. The $\Gamma_{j}$ can be chosen so that the measure $\mu$ which is $d z$ on $\Gamma_{0}$ and $-d z$ on $\Gamma_{j}, 1 \leqq j<\infty$, is a finite measure. The measure $\mu$ satisfies

$$
\begin{array}{ll}
\int f d \mu=0, & f \in R(X) \\
\int f d \mu=2 \pi i f^{\prime}(\infty), & f \in B_{\Gamma} .
\end{array}
$$

The mass of $\mu$ is carried on $X^{\circ}$. It follows from Lebesgue's dominated convergence theorem that no function $f \in B_{\Gamma}$ satisfying $f^{\prime}(\infty) \neq 0$ can be a pointwise limit on $X^{\circ}$ of a bounded sequence in $R(X)$. In particular, we obtain

(6) $R(X)$ is not pointwise boundedly dense in $H^{\infty}\left(X^{\circ}\right)$.

6. Vector valued approximation. In this section we note that Vitushkin's techniques for rational approximation extend to vector valued analytic functions. This observation leads to results on an old problem about tensor products. The results of this section are motivated by Eifler's paper [7].

Let $X$ be a compact plane set, and let $W$ be a Banach space. Let $C(X, W)$ denote the Banach space of continuous functions from $X$ to $W$, with the usual supremum norm. Let $A(X, W)$ be the subspace of $C(X, W)$ of functions analytic on $X^{\circ}$, and let $R(X, W)$ be the closure in $C(X, W)$ of the functions which extend to be analytic in a neighborhood of $X$.

By Dugundji's theorem, we may assume that each function in $C(X, W)$ is extended to the complex plane, has compact support, and attains its norm on $X$. The modulus of continuity of the extended functions will be denoted by $\omega$.

6.1 THEOREM. The following are equivalent, for $f \in C(X, W)$ :

(a) $f \in R(X, W)$.

(b) For each $L \in W^{*}, L \circ f \in R(X)$. 
(c) For every infinitely differentiable function $\phi$ supported on any disc $\Delta\left(z_{0} ; \delta\right)$,

$$
\left\|\iint f(z) \frac{\partial \phi}{\partial \bar{z}} d x d y\right\| \leqq 2 \pi \delta \omega(f ; 2 \delta)\left\|\frac{\partial \phi}{\partial \bar{z}}\right\|_{\infty} \alpha\left(\Delta\left(z_{0} ; \delta\right) \backslash K\right) .
$$

(d) There exist $r \geqq 1$, and a function $\Omega(\delta) \rightarrow 0$ as $\delta \rightarrow 0$, such that for any infinitely differentiable function $\phi$ supported on any disc $\Delta\left(z_{0} ; \delta\right)$,

$$
\left\|\iint f(z) \frac{\partial \phi}{\partial \bar{z}} d x d y\right\| \leqq \delta \Omega(\delta)\left(\left\|\frac{\partial \phi}{\partial x}\right\|_{\infty}+\left\|\frac{\partial \phi}{\partial y}\right\|_{\infty}\right) \alpha\left(\Delta\left(z_{0} ; r \delta\right) \backslash K\right) .
$$

Proof. This theorem is proved for scalar valued functions by Vitushkin [13]. The proof of the general case involves only a slight modification of Vitushkin's work. We will only sketch the necessary changes.

It is clear that (a) implies (b), and that (c) implies (d). That (b) implies (c) is essentially Lemma IV.2.2 of [13], in the case of scalar valued functions. The vector valued case follows from the scalar valued case by taking suprema over linear functionals on $W$.

Finally, (d) implies (a), by the arguments developed in sections IV.3 and IV.4 of [13], with only two minor changes. Absolute value signs must be replaced by norms at the appropriate places. And the coefficient matching Lemma IV.3.2 must be replaced by the following statement: If $E$ is a subset of the complex plane with analytic center $z_{0}$, and if $a, b \in W$ satisfy $\|a\| \leqq \alpha(E)$ and $\|b\| \leqq \beta(E)$, then there is $f \in C\left(S^{2}, W\right)$ such that $\|f\|_{\infty} \leqq 6, f$ is analytic off a compact subset of $E$, and

$$
f(z)=\frac{a}{z-z_{0}}+\frac{b}{\left(z-z_{0}\right)^{2}}+O\left(\frac{1}{\left|z-z_{0}\right|^{3}}\right)
$$

near $\infty$. The proof of this latter statement is exactly the same as that of the scalar version in [13].

6.2 Corollary. If $R(X)=A(X)$, then $R(X, W)=A(X, W)$ for all Banach spaces $W$.

Now suppose that $X$ and $Y$ are compact spaces, and that $A$ and $B$ are closed subspaces of $C(X)$ and $C(Y)$ respectively. The tensor product $A \otimes B$ of $A$ and $B$ is the closed linear span in $C(X \times Y)$ of functions of the form $f(x) g(y)$, where $f \in A$ and $g \in B$. The slice subspace $S$ of $C(X \times Y)$ consists of $G \in C(X \times Y)$ such that $y \rightarrow G(x, y)$ belongs to $B$ for all fixed $x \in X$, and $x \rightarrow G(x, y)$ belongs to $A$ for all fixed $y \in Y$. Clearly $A \otimes B \subseteq S$. If $A$ and $B$ are algebras, then so are $A \otimes B$ and $S$. It is not known whether $A \otimes B$ and $S$ must always coincide.

It is natural to identify $C(X \times Y)$ with $C(X, C(Y))$. Under this identification, $S$ becomes the set of all $f \in C(X, B)$ such that $L \circ f \in A$ for all linear functionals $L$ on $B$. This follows, for instance, from a simple argument using the Riesz representation theorem and the Hahn-Banach theorem. 
Now suppose that $X$ is a plane set. Then $R(X) \otimes B$ can be identified with $R(X, B)$. In view of 6.1 , we obtain the following.

6.3 COROLlaRY. If $X$ is a compact plane set, $Y$ is a compact Hausdorff space, and $B$ is a closed subspace of $C(Y)$, then $R(X) \otimes B$ coincides with the slice subspace $S$.

By $P(X)$ we denote the closure in $C(X)$ of the polynomials in $z$. By Mergelyan's theorem, $P(X)=A(X)$ whenever $X$ has a connected complement. If $X$ and $Y$ are compact plane sets with connected complements, then it seems eminently reasonable that also $P(X \times Y)=A(X \times Y)$. We know of no elementary proof of this fact (based on Mergelyan's theorem). However, that the theorem is true follows by taking $B=A(Y)$ in 2.3 , and noting that $P(X \times Y)=R(X \times Y)$ in this case. More generally, we have the following

6.4 Corollary. If $X$ is a compact plane set such that $R(X)=A(X)$, and if $Y$ is a compact subset of $C^{n}$ such that $R(Y)=A(Y)$, then $R(X \times Y)=A(X \times Y)$.

\section{REFERENCES}

1. P. Ahern and D. Sarason, On some hypodirichlet algebras of analytic functions, Amer. J. Math. 89 (1967), 932-941.

2. R. Arens, The maximal ideals of certain function algebras, Pacific J. Math. 8 (1958), 641-648.

3. A. Browder and J. Wermer, Some algebras of functions on an arc, J. Math. Mech. 12 (1963), 119-130.

4. P. C. Curtis, Jr., Peak points for algebras of analytic functions, J. Functional Analysis 3 (1969), 35-47.

5. E. P. Dolzhenko, On approximation on closed regions and on nullsets, Dokl. Akad. Nauk SSSR 143 (1962), 771-774=Soviet Math. Dokl. 3 (1962), 472-475.

6. J. Dugundji, An extension of Tietze's theorem, Pacific J. Math. 1 (1951), 353-367.

7. L. Eifler, The approximation property for some function algebras (to appear).

8. S. Fisher, Bounded approximation by rational functions, Pacific J. Math. 28 (1969), 319-326.

9. T. Gamelin, Uniform algebras, Prentice-Hall, Englewood Cliffs, N. J., 1969.

10. J. Garnett, On a theorem of Mergelyan, Pacific J. Math. 26 (1968), 461-467.

11. I. Glicksberg, Dominant representing measures and rational approximation, Trans. Amer. Math. Soc. 130 (1968), 425-462.

12. L. Ruble and A. Shields, Bounded approximation by polynomials, Acta Math. 112 (1964), $145-162$.

13. A. G. Vitushkin, Analytic capacity of sets and problems in approximation theory, Uspehi Mat. Nauk 22 (1967), 141-199.

14. J. Wermer, Seminar über Funktionen-Algebren, Lecture Notes in Math. No. 1, SpringerVerlag, Berlin, 1964.

15. L. Zalcman, Analytic capacity and rational approximation, Lecture Notes in Math. No. 50, Springer-Verlag, Berlin, 1968.

16. - Bounded analytic functions on infinitely connected domains, Trans. Amer. Math. Soc. (to appear).

UNIVERSiTy OF CALIFORNia, Los ANGeles, CALIForNia 